\title{
Long-Term Stroke Recurrence after Transient Ischemic Attack: Implications of Etiology
}

\author{
Angel Ois, ${ }^{\text {a,b,c,d }}$ Elisa Cuadrado-Godia, ${ }^{\mathrm{a}, \mathrm{b}, \mathrm{c}, \mathrm{d}}$ Eva Giralt-Steinhauer, ${ }^{\mathrm{a}, \mathrm{b}, \mathrm{c}, \mathrm{d}}$ Jordi Jimenez-Conde, ${ }^{\mathrm{a}, \mathrm{b}, \mathrm{c}, \mathrm{d}}$ \\ Carolina Soriano-Tarraga, ${ }^{\mathrm{a}, \mathrm{b}, \mathrm{c}, \mathrm{d}}$ Ana Rodríguez-Campello, ${ }^{\mathrm{a}, \mathrm{b}, \mathrm{c}, \mathrm{d}}$ Carla Avellaneda, ${ }^{\mathrm{a}, \mathrm{b}, \mathrm{c}, \mathrm{d}}$ Diego Cascales, ${ }^{\mathrm{a}}$ \\ Isabel Fernandez-Perez, ${ }^{\mathrm{a}}$ Jaume Roquer ${ }^{\mathrm{a}, \mathrm{b}, \mathrm{c}, \mathrm{d}}$ \\ aDepartment of Neurology, Hospital del Mar, Barcelona, Spain \\ ${ }^{b}$ Neurovascular Research Group, IMIM (Institut Hospital del Mar d'Investigacions Mèdiques), Barcelona, Spain \\ 'Autonomous University of Barcelona, Barcelona, Spain \\ ${ }^{d}$ Department of Experimental and Health Sciences, University Pompeu Fabra, Barcelona, Spain
}

Background and Purpose To analyze long-term stroke recurrence (SR) characteristics after transient ischemic attack (TIA) according to initial etiological classification.

Methods A prospective cohort of 706 TIA patients was followed up in a single tertiary stroke center. Endpoint was SR. Etiologic subgroup was determined according to the evidence-based causative classification system. Location of TIA and SR was recorded as right, left, or posterior territory. Disability stroke recurrence (DSR) was defined as modified Rankin Scale (mRS) score $>1$ or a onepoint increase in those with previous $\mathrm{mRS}>1$ at 3-month follow-up.

Results During a follow-up of 3,493 patient-years (mean follow-up of $58.9 \pm 35.9$ months), total SR was $125(17.7 \%)$, corresponding to 3.6 recurrences per 100 patient-years. The etiology subgroups with a higher risk of SR were the unclassified (more than one cause) and large-artery atherosclerosis (LAA) categories. Of the SR cases, 88 (70.4\%) had the same etiology as the index TIA; again, LAA etiology was the most frequent (83.9\%). Notably, cardioaortic embolism was the most frequent cause (62.5\%) of SR in the subgroup of 24 patients with undetermined TIA. Overall, SR occurred in the same territory in 74 of 125 patients (59.2\%), with significant differences between etiological TIA subgroups ( $P=0.015)$. Eighty-two of $125(65.6 \%)$ with SR had DSR, without differences between etiologies $(P=0.453)$.

Conclusions SR occurred mainly with the same etiology and location as initial TIA, although undetermined TIA was associated with a high proportion of cardioaortic embolism SR. More than half of the recurrences caused some disability, regardless of etiology.

Keywords Stroke; Recurrence; Ischemic attack, transient
Correspondence: Eva Giralt-Steinhauer Department of Neurology, Hospital del Mar, Passeig Marítim 25-29, Barcelona 08003, Spain

Tel: $+34-932483235$

Fax: $+34-932483615$

E-mail: egiralt@imim.es

Received: December 24, 2018

Revised: January 23, 2019

Accepted: February 18, 2019

\section{Introduction}

Assessing the early risk of stroke recurrence (SR) after transient ischemic attack (TIA) has been the goal of many studies. ${ }^{1}$ However, few reports have analyzed data related to long-term SR after TIA. ${ }^{2-7}$ It is usually assumed that the etiologic cause of TIA and early recurrence are the same, but in prolonged follow-up this may not be the case. Consequently, the relationship of SR etiology to the initial TIA has not been described. We hypothesized that the etiology of TIA can be related to the cause, loca- 
tion, and prognosis of long-term SR. Our objective was to describe the long-term SR characteristics (etiology, location, and outcome) after a TIA and relate them to the etiological data recorded at the time of TIA diagnosis.

\section{Methods}

A prospective observational registry was designed, including all consecutive patients with first-ever TIA evaluated in our stroke unit within the first 24 hours of symptoms onset from January 2005 to June 2016 ( $n=706)$. Hospital del Mar is a tertiary stroke center with an on-call neurologist and a stroke unit, as well as capabilities for systemic thrombolysis and neurovascular interventions. In its catchment area, which comprises the 330,000 inhabitants of three of the 10 city districts of Barcelo$n a$, it is the only hospital that can provide urgent etiological studies in patients with TIA. ${ }^{8}$ According to World Health Organization criteria, TIA was defined as rapidly developing clinical symptoms and/or signs of focal loss of cerebral function with reversal symptoms within 24 hours (classical criteria ${ }^{9}$ ) with no apparent cause other than of vascular origin. ${ }^{10}$ All patients with alternative non-vascular explanation of symptoms (TIA mimics, $n=16)$, those not evaluated in the stroke unit $(n=9)$, and those with incomplete data or who declined study inclusion $(n=7)$ were excluded. Follow-up consisted of a visit with a neurologist at 3 months post-TIA and, at the physician's discretion, a clinic appointment and/or telephone contact every 3 to 6 months thereafter until study conclusion (December 2017); 227 patients were censored due to losses to follow-up ( $n=34$, $4.8 \%)$ and non-neurological death $(n=168,23.8 \%)$. We reviewed all patient events, deaths, electronic medical records, and hospital admissions records, and consulted the primary care physician before considering patients lost to follow-up. This methodology has been detailed in a previous study."

\section{Data collection and work-up}

A structured questionnaire was used to record age, sex, and vascular risk factors, defined according to previously reported criteria: hypertension, diabetes mellitus, hyperlipidemia, current smoking habit, and atrial fibrillation. ${ }^{11,12}$ We also recorded the following data: previous TIA, defined as a TIA in the 7 days preceding the index TIA, ABCD2 ${ }^{1}$ score and multiple TIA defined as new TIA within 72 hours of admission for index TIA. Based on all available data at hospital discharge (clinical data and explorations), we assigned one etiology subgroup for each TIA following an evidence-based causative classification system (CSS) for ischemic stroke: large-artery atherosclerosis (LAA), small-artery disease (SAD), cardioaortic embolism (CE), unde- termined causes, unclassified strokes (more than one apparent mechanism), and other (infrequent) causes. ${ }^{13}$ We applied the SAD classification when patients with no evidence of atherothrombotic or CE cause reported classical lacunar syndrome (pure motor, pure sensitive, and sensitive-motor syndrome involving at least two of three specific body parts [face, arm, and leg] and ataxic hemiparesis or dysarthria-clumsy hand syndrome). ${ }^{14}$ Patients with no evidence of atherothrombotic or CE cause who reported cortical symptoms were assigned the "undetermined" classification. All patients (in both TIA and SR events, except when the responsible physician considered a short time had elapsed between the two) were assessed in the same way by a vascular-trained neurologist following our group's previously described therapeutic and diagnostic workup. ${ }^{11,12}$ Briefly, this includes a complete neurological and physical exploration, blood test, 12-lead electrocardiography, brain computed tomography (CT), and neurovascular imaging (magnetic resonance imaging, $\mathrm{CT}$ and/or ultrasound that includes the study of supra-aortic trunks and intracranial territory). After hospital admission, all patients had continuous electrocardiography monitoring in the stroke unit for at least 24 hours. When no etiologic cause was determined, echocardiography (transthoracic and/or transesophageal) was performed. Continuous Holter monitoring ( 24 hours or 7 to 20 days) or implantable cardiac device also was performed after hospital discharge, but this information was not used to determine initial etiology. As part of our work routine, the clinical data associated with the TIA are thoroughly explored with patients, relatives, or witnesses. Acute infarction $(n=129)$ was detected by CT, performed in all 706 patients (although images for 27 patients were not available for analysis), and/or by magnetic resonance studies (available for 357 patients [50.6\%]). Based on these data, the location of the symptoms was categorized as right, left, or posterior (typical symptoms of vertebrobasilar arteries: ${ }^{15}$ e.g., diplopia, ataxia, isolated vision loss in campimetry). Due to the lack of information about isolated dysarthria localization, the present study did not consider this as a TIA. Following recommendations of appropriate guidelines, 537 patients were treated with antiplatelet (single or double) plus statins, except those that received anticoagulation therapy: 163 patients with major CE source, two with other infrequent causes, and two with multiple TIA despite antiplatelet therapy. Carotid surgery $(n=40)$ or arterial stenting $(n=8$, three out of internal carotid artery) was performed within 20 days from TIA onset in all patients with clinical criteria except one patient who refused treatment. A strict control of all vascular risk factors, appropriate diet, and physical exercise was recommended in all patients. 


\section{Endpoint}

The primary study endpoint was $S R$, defined as a new neurological event with symptoms duration $\geq 24$ hours and acute radiological lesion (ischemic or hemorrhagic) attributable to symptoms. Secondary outcomes were: disability stroke recurrence (DSR), defined as modified Rankin Scale $(\mathrm{mRS})^{16}$ score $>1$ or a one-point increase in those with previous MRS $>1$ at 3-month follow-up and localization of SR same/different territory categorized as right, left, or posterior.

\section{Ethics}

The local ethics committee approved the register. We obtained written informed consent from all participants or a designated representative, as appropriate.

\section{Statistical analysis}

Descriptive data are presented as mean \pm SD and as a number and percentage for categorical variables. Time-data are expressed in months. All comparative analysis are time-depended and were obtained by Kaplan-Meier log rank test. Statistical analyses were performed with the SPSS version 23.0 for Windows (IBM Co., Armonk, NY, USA). Statistical analysis was reviewed by Eva Giralt-Steinhauer, Specialization in Statistics Applied to Health Sciences (Universitat Autònoma de Barcelona).

\section{Results}

The study sample was 706 patients, with a mean age of $71.4 \pm 13.9$ years. Mean follow-up was $58.9 \pm 35.9$ months (range, 0 to $148 ; 3,493$ patient-years). Demographics and vascular risk factors are shown in Table 1. The largest etiological subgroup of index TIA was SAD (29.1\%), followed by undetermined cause (26.9\%) and CE (21.4\%). There were 125 patients with SR (17.7\%; 3.6 recurrences per 100 patient-years) and seven patients (1\%) had more than one SR during follow-up. The subgroups with a higher risk of SR were unclassified etiology (11/24) and LAA (31/126); undetermined and other less frequent causes showed lower SR risk. Regarding the SR etiology, 88 of 125 patients (70.4\%) had the same etiology as their index TIA, with LAA the most frequent category, followed by unclassified and CE. We found that 37 of 125 patients (29.6\%) had a different etiology in the SR, mainly in the initially undetermined subgroup, in which SR etiology was different in 16 of 24 (66.6\%), of whom $62.5 \%$ were CE. Nine patients $(7.2 \%)$ had hemorrhagic recurrence, mainly from SAD TIA subgroup $(n=4,44.4 \%)$. Other SR etiologies that differed from the initial TIA etiology were much less frequent (Table 2). A DSR occurred in $82(65.6 \%)$ of
Table 1. Baseline characteristics

\begin{tabular}{lc}
\hline Variable & Total $(\mathrm{n}=706)$ \\
\hline Age $(\mathrm{yr})$ & $71.4 \pm 13.9$ \\
Male sex & $372(52.7)$ \\
Hypertension & $509(72.1)$ \\
Diabetes mellitus & $194(27.5)$ \\
Hyperlipidemia & $353(50)$ \\
Current smoking & $68(9.6)$ \\
Atrial fibrillation & $163(23.1)$ \\
Previous TIA & $139(19.7)$ \\
Multiple TIA* & $62(8.8)$ \\
ABCD 2 risk & \\
Low & $203(28.8)$ \\
Moderate & $384(54.4)$ \\
High & $119(16.9)$ \\
\hline
\end{tabular}

Values are presented as mean \pm standard deviation or number $(\%)$. TIA, transient ischemic attack.

${ }^{*}$ Multiple TIA: new TIA within 72 hours of admission for TIA index; ${ }^{+}$ABCD2 score, stratified as 0-3 (low risk), 4-5 moderate, $>5$ high.

SR patients, without significant differences $(P=0.453)$ between etiological categories. Excluding patients lost to follow-up, 57 patients (46\%) with SR died, compared with 136 patients $(24.8 \%)$ without SR $(P<0.001)$ (Table 3). Regarding localization, 74 patients $(59.2 \%)$ had a SR within the same territory as the index TIA, with significant differences between etiological SR subgroups (higher rates in $L A A ; P=0.010$ ). Temporal description of $\mathrm{SR}$ characteristic is detailed in Figure 1. SR onset was significantly later in those patients with SR etiology different from the index TIA ( $35 \pm 35.3$ months vs. $12.1 \pm 22.3$ months, $P<0.001)$ but earlier in those with the same affected territory $(12 \pm 25.8$ months vs. $28.8 \pm 29.8$. months, $P<0.001)$.

\section{Discussion}

Few studies have shown the long-term risk of SR after TIA and results differ depending on study design (population, national registers, hospital registers) and specific objectives. ${ }^{2-7}$ The SR risk in our study was in the low range reported by previous studies and consistent with recent data that show a lower risk of SR in patients who are treated urgently in stroke units and in whom the etiological study is performed immediately. ${ }^{7,17}$

To our knowledge, this is the first study to analyze the relationship between the index TIA and long-term SR etiology. These data provide relevant knowledge for clinical practice involving these patients. We observed that SR occurs more frequently in subgroups associated with significant atherosclerosis (LAA and unclassified [more than one major cause]). This 
Table 2. Etiological classification of stroke recurrence and relationship with index TIA etiological subgroups

\begin{tabular}{|c|c|c|c|c|c|c|}
\hline \multirow{2}{*}{ Variable } & \multicolumn{6}{|c|}{ TIA etiology } \\
\hline & SAD & Undetermined & CE & LAA & Unclassified & Other \\
\hline Total population & $199(29.1)$ & $190(26.9)$ & $151(21.4)$ & $126(17.8)$ & $24(3.4)$ & $16(2.3)$ \\
\hline Stroke recurrence (\% each subgroup) & $30(15.1)$ & $24(12.6)$ & $27(17.9)$ & $31(24.6)$ & $11(45.8)$ & $2(12.5)$ \\
\hline Same etiology & $21(70)$ & $8(33.3)$ & $22(81.5)$ & $26(83.9)$ & $9(81.8)$ & $2(100)$ \\
\hline \multicolumn{7}{|l|}{ Different etiology } \\
\hline SAD & - & $1(4.1)$ & - & - & - & - \\
\hline Undetermined & $2(6.6)$ & - & - & - & - & - \\
\hline CE & $3(10)$ & $15(62.5)$ & - & - & $2(18.2)$ & - \\
\hline LAA & - & - & - & - & - & - \\
\hline Unclassified & - & - & $2(7.4)$ & $1(3.2)$ & - & - \\
\hline Other less frequent & - & - & - & $2(6.4)$ & - & - \\
\hline Hemorrhagic & $4(13.3)$ & - & $3(11.1)$ & $2(6.4)$ & - & - \\
\hline
\end{tabular}

Values are presented as number (\%).

TIA, transient ischemic attack; SAD, small-artery disease; CE, cardioaortic embolism; LAA, large-artery atherosclerosis.

Table 3. Data about location (same/not same vascular territory) and prognosis (disabling/fatal) of stroke recurrence, related to index transient ischemic attack etiologies

\begin{tabular}{lcccccccc}
\hline \multirow{2}{*}{ Variable } & Total $(n=125)$ & \multicolumn{5}{c}{ Stroke recurrence etiology } \\
\cline { 3 - 7 } & & SAD $(n=30)$ & Undetermined $(n=24)$ & CE $(n=27)$ & LAA $(n=31)$ & Unclassified $(n=11)$ & Other $(n=2)$ & $P$ \\
\hline Same location & $74(59.2)$ & $19(63.3)$ & $9(37.5)$ & $12(44.4)$ & $25(80.6)$ & $8(72.7)$ & $1(50)$ & 0.015 \\
Disabling/fatal & $82(65.6)$ & $20(66.7)$ & $14(58.3)$ & $18(66.7)$ & $24(77.4)$ & $5(45.5)$ & $1(50)$ & 0.453 \\
\hline
\end{tabular}

Values are presented as number (\%).

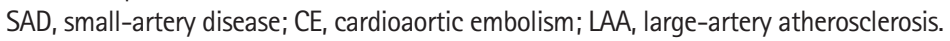
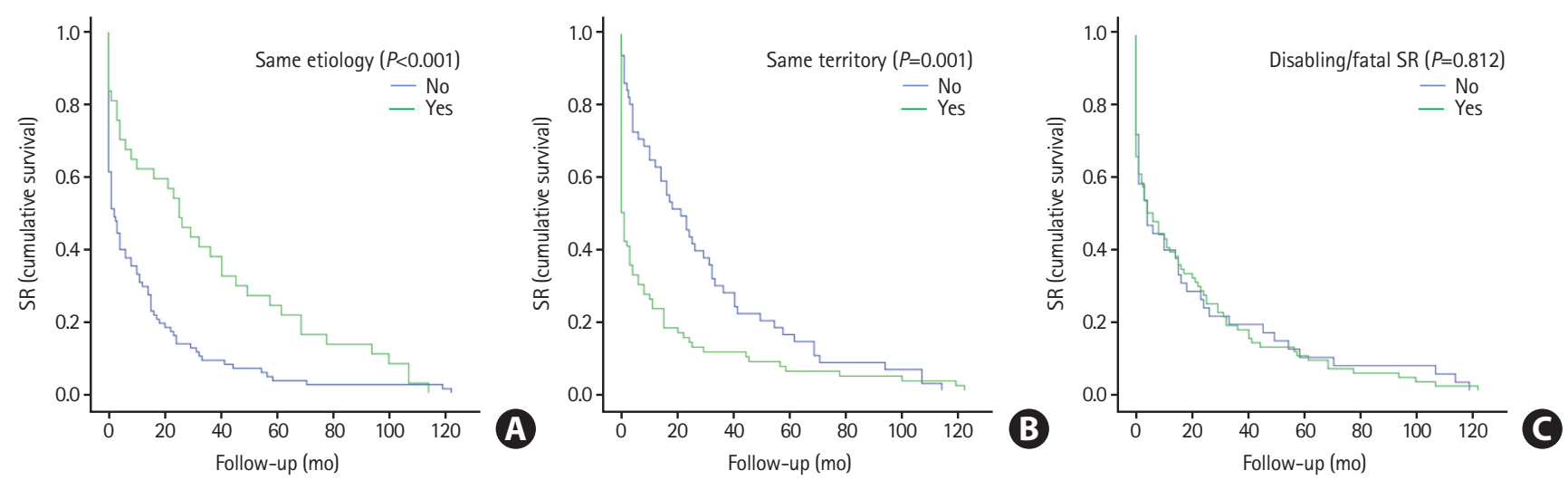

Figure 1. Temporal description of characteristics of stroke recurrence (SR). (A) According to etiology. (B) According to vascular territory. (C) According to prognosis.

relationship between etiological subgroup and the risk of recurrence has been previously described ${ }_{1}^{12}$ being LAA the main established predictor of subsequent early stroke. ${ }^{8,18}$ Our data show that the majority of SR events have the same etiology as the index TIA. The proportion is higher in the unclassified, LAA, and CE subgroups, likely due to the difficulty of resolving the underlying causes that increase the risk of SR (arterial atherosclerosis and/or structural heart disease). Nonetheless, we detected a difference in etiology between the index TIA and SR in almost 30\% of patients, especially in those with later SR. In 
the undetermined TIA subgroup, most of the SR had CE etiology due to AF detection or development of other major CE causes over the years. These findings are relevant because patients with undetermined TIA are screened for CE sources ${ }^{19,20}$ and treated according to the current recommendations. Although a low rate of AF detection in TIAs has been reported, ${ }^{21}$ our results suggest a subgroup of patients (cortical symptoms without a major cause) that could benefit more from prolonged cardiac monitoring strategies. The second cause of diverse etiology in the SR was hemorrhagic stroke, which occurred more frequently in patients with TIA of SAD etiology; these entities have similar pathophysiological mechanisms. ${ }^{22}$ The Secondary Prevention of Small Subcortical Strokes (SPS3) trial study, one of the main studies of patients with SAD, reported higher hemorrhagic risk in these patients and emphasized that control of hypertension is as important as antithrombotic treatment. ${ }^{23}$ The remaining etiological groups with hemorrhagic SR contain patients with higher risk for hemorrhagic strokes due to more aggressive antithrombotic treatments, such as CE and LAA etiologies. Other etiologies that differed between SR and index TIA showed marginal percentages in our study.

Our data showed that most events occurred in the same location as the initial TIA. This is expected in patients with etiologies associated with significant atherosclerosis (LAA and unclassified), although not in patients with CE or SVD etiology. However, the neurologist does not usually see the symptoms in patients with TIA because these have been resolved upon arrival at the hospital; therefore, we could only categorize them on the affected side or as vertebral basilar symptoms, with the limitations that this supposed for the interpretation of this finding.

Regarding stroke outcome, an important percentage of patients had a DSR event and decreased survival. However, to compare our results with similar studies, we must take into account that we used a very restrictive DSR definition $(m R S>1)$. Our data highlight the importance of secondary prevention in these patients and the need of further analyzing the etiological mechanisms at the time of $S R$ and determining how they relate to the initial etiology of the TIA. New therapeutic strategies, including new anticoagulants, treatment with double antiplatelets in the first months after TIA, or new treatments in refractory hyperlipidemia, could reduce this risk in these patients with SR of the same etiology. It was not unexpected that SR with different etiology from the index TIA occurred later, compared to recurrence at the same location, although this observation had not been previously reported. However, there is a need for further studies with more patients in each etiological group and radiological data providing more information on the localization of symptoms in order to ensure a correct interpre- tation of our observation and to establish its usefulness.

Strengths of the study were that a long-term cohort of patients was directly evaluated by neurologists experienced in vascular pathology and admitted to the stroke unit with cardiac monitoring. The study had several limitations in addition to those already considered throughout the discussion. We assessed a relatively small number of patients and the sample was drawn from a hospital cohort; therefore, our results cannot be extrapolated to other cohorts. Our cohort had a high mean age with relatively high all-cause mortality. All patients received the most appropriate secondary prevention treatments according to current guidelines but we did not have data on adjustments over the years in response to new pathologies. Finally, we used a clinical TIA definition and did not routinely perform a magnetic resonance imaging study at hospital admission, so we cannot analyze the existence of acute lesions on diffusion-weighted imaging. These limitations do not invalidate the conclusions of the study.

\section{Conclusions}

Almost one in five patients had a long-term SR after a first TIA, and these events were mainly related to atherosclerotic and $\mathrm{CE}$ origins. In most cases, the recurrences had the same etiology as the index TIA; the exception occurred in initial TIA of undetermined etiology, with recurrences mostly classified as CE strokes. In our study, most patients had a DSR, highlighting the importance of secondary prevention.

\section{Disclosure}

The authors have no financial conflicts of interest.

\section{Acknowledgments}

Supported in part by Spain's Ministry of Health (Ministerio de Sanidad y Consumo, Instituto de Salud Carlos III FEDER, RD12/0042/0020 INVICTUS-PLUS).

Elaine M. Lilly, PhD, provided English language assistance.

\section{References}

1. Johnston SC, Rothwell PM, Nguyen-Huynh MN, Giles MF, Elkins JS, Bernstein AL, et al. Validation and refinement of scores to predict very early stroke risk after transient ischaemic attack. Lancet 2007;369:283-292.

2. Wardlaw JM, Brazzelli M, Chappell FM, Miranda H, Shuler K, Sandercock PA, et al. ABCD2 score and secondary stroke pre- 
vention: meta-analysis and effect per 1,000 patients triaged. Neurology 2015;85:373-380.

3. Guarino $M$, Rondelli $F$, Favaretto $E_{1}$ Stracciari $A$, Filippini $M_{1}$ Rinaldi $R$, et al. Short- and long-term stroke risk after urgent management of transient ischaemic attack: the Bologna TIA Clinical Pathway. Eur Neurol 2015;74:1-7.

4. Holzer K, Feurer R, Sadikovic S, Esposito L, Bockelbrink $A$, Sander $D$, et al. Prognostic value of the ABCD2 score beyond short-term follow-up after transient ischemic attack (TIA): a cohort study. BMC Neurol 2010;10:50.

5. Kiyohara T, Kamouchi M, Kumai Y, Ninomiya T, Hata J, Yoshimura $S$, et al. ABCD3 and ABCD3-I scores are superior to $A B C D 2$ score in the prediction of short- and long-term risks of stroke after transient ischemic attack. Stroke 2014;45:418425.

6. van Wijk I, Kappelle U, van Gijn J, Koudstaal PJ, Franke CL, Vermeulen $\mathrm{M}$, et al. Long-term survival and vascular event risk after transient ischaemic attack or minor ischaemic stroke: a cohort study. Lancet 2005;365:2098-2104.

7. Amarenco $P$, Lavallée PC, Monteiro Tavares L, Labreuche J, Albers GW, Abboud $H$, et al. Five-year risk of stroke after TIA or minor ischemic stroke. N Engl J Med 2018;378:2182-2190.

8. Amarenco P, Lavallée PC, Labreuche J, Albers GW, Bornstein $N M$, Canhão $P$, et al. One-year risk of stroke after transient ischemic attack or minor stroke. N Engl J Med 2016;374: 1533-1542.

9. Albers GW, Caplan LR, Easton JD, Fayad PB, Mohr JP, Saver $J$, et al. Transient ischemic attack: proposal for a new definition. N Engl J Med 2002;347:1713-1716.

10. Aho K, Harmsen P, Hatano S, Marquardsen J, Smirnov VE, Strasser T. Cerebrovascular disease in the community: results of a WHO collaborative study. Bull World Health Organ 1980;58:113-130.

11. Ois A, Zabalza A, Moreira A, Cuadrado-Godia E, Jimenez-

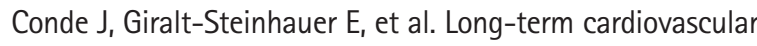
prognosis after transient ischemic attack: associated predictors. Neurology 2018;90:e553-e558.

12. Ois $A$, Gomis $M$, Rodríguez-Campello A, Cuadrado-Godia $E_{1}$

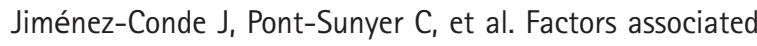
with a high risk of recurrence in patients with transient ischemic attack or minor stroke. Stroke 2008;39:1717-1721.

13. Ay $H$, Furie $K L$, Singhal $A$, Smith WS, Sorensen AG, Koroshetz
WJ. An evidence-based causative classification system for acute ischemic stroke. Ann Neurol 2005;58:688-697.

14. Hervé $D$, Gautier-Bertrand $M$, Labreuche J, Amarenco P; GENIC Investigators. Predictive values of lacunar transient ischemic attacks. Stroke 2004;35:1430-1435.

15. Markus HS, van der Worp HB, Rothwell PM. Posterior circulation ischaemic stroke and transient ischaemic attack: diagnosis, investigation, and secondary prevention. Lancet Neurol 2013;12:989-998.

16. Banks JL, Marotta CA. Outcomes validity and reliability of the modified Rankin Scale: implications for stroke clinical trials: a literature review and synthesis. Stroke 2007;38:1091-1096.

17. Kennedy J, Hill MD, Ryckborst KJ, Eliasziw M, Demchuk AM, Buchan AM, et al. Fast assessment of stroke and transient ischaemic attack to prevent early recurrence (FASTER): a randomised controlled pilot trial. Lancet Neuro/ 2007;6:961969.

18. Purroy F, Montaner J, Molina CA, Delgado P, Ribo M, AlvarezSabín J. Patterns and predictors of early risk of recurrence after transient ischemic attack with respect to etiologic subtypes. Stroke 2007;38:3225-3229.

19. Sanna T, Diener HC, Passman RS, Di Lazzaro V, Bernstein RA, Morillo $C A$, et al. Cryptogenic stroke and underlying atrial fibrillation. N Engl J Med 2014;370:2478-2486.

20. Vivanco Hidalgo RM, Rodríguez Campello A, Ois Santiago Á, Cuadrado Godia E, Pont Sunyer C, Roquer J. Cardiac monitoring in stroke units: importance of diagnosing atrial fibrillation in acute ischemic stroke. Rev Esp Cardiol 2009;62:564567.

21. Korompoki E, Del Giudice A, Hillmann S, Malzahn U, Gladstone DJ, Heuschmann $\mathrm{P}$, et al. Cardiac monitoring for detection of atrial fibrillation after TIA: a systematic review and meta-analysis. Int J Stroke 2017;12:33-45.

22. Marsh EB, Gottesman RF, Hillis AE, Maygers J, Lawrence $E_{1}$ Llinas $\mathrm{RH}$. Predicting symptomatic intracerebral hemorrhage versus lacunar disease in patients with longstanding hypertension. Stroke 2014;45:1679-1683.

23. SPS3 Study Group, Benavente OR, Coffey CS, Conwit R, Hart RG, McClure LA, et al. Blood-pressure targets in patients with recent lacunar stroke: the SPS3 randomised trial. Lancet 2013;382:507-515. 